\title{
A new high-quality scholarly journal will help drive physiotherapy towards being an evidence-based healthcare profession in France
}

\author{
Mark R. Elkins ${ }^{1,2,3}$, Anne M. Moseley ${ }^{4}$ and Rafael Zambelli Pinto $3,5,6$ \\ ${ }^{1}$ Centre for Education and Workforce Development, Sydney Local Health District, Sydney, Australia, ${ }^{2}$ Faculty of Medicine and Health, The University of Sydney, \\ Sydney, Australia, ${ }^{3}$ International Society of Physiotherapy Journal Editors, ${ }^{4}$ Institute for Musculoskeletal Health, The University of Sydney and Sydney Local \\ Health District, Sydney, Australia, ${ }^{5}$ Department of Physical Therapy, Federal University of Minas Gerais (UFMG), Belo Horizonte, MG, Brazil, ${ }^{6}$ Sydney School of \\ Public Health, Faculty of Medicine and Health, The University of Sydney, Sydney, Australia.
}

DOI: $10.52057 /$ erj.v1i1.1

received : 11 November 2020

accepted: 3 February 2021

\begin{abstract}
7 his editorial has several purposes. It reviews the importance of establishing and maintaining a high quality, local, research journal as a forum to both foster and demonstrate a research culture among a nation's physiotherapists. It welcomes European Rehabilitation Journal the journal of the Société Française de Physiothérapie. It also commends the other initiatives and achievements of the Société Française de Physiothérapie towards establishing physiotherapy as an evidence-based healthcare profession in France. Finally, it recognises the profession's need and readiness for the training of physiotherapists in France to progress to a university degree course.
\end{abstract}

It may surprise some readers to learn that the International Society of Physiotherapy Journal Editors has around 130 member journals, many of which are journals linked to a national physiotherapy member association [1]. However, physiotherapy research is not just published in physiotherapy-specific journals. For example, clinical trials of the effects of physiotherapy interventions have been published in over 4,300 journals as diverse as Breast Cancer Research and Treatment, Critical Care Medicine, Spinal Cord, World Journal of Urology, Obesity Research and Developmental Medicine and Child Neurology. These many forums for publication of physiotherapy research raise the question: is another journal needed? We strongly contend that it is. Establishing and maintaining a high quality, local, research journal has many advantages for the physiotherapy profession in France. It promotes a culture of research and emphasises the need for evidence-based practice by the profession. It provides a key forum for disseminating research conducted locally and for raising professional issues that have implications for local clinical practice. It provides opportunities to gain experience in conducting, reviewing and editing research. It also allows an international visibility for French physiotherapy research that has often been hidden behind the medical profession. Notably, these advantages all contribute to a form of emancipation for the physiotherapy profession in France.

Mark R. Elkins, Centre for Education and Workforce Development, Sydney Local Health District, Australia mail : mark.elkins@sydney.edu.au ORCiD:

0000-0002-2052-7366
We commend the Société Française de Physiothérapie for establishing European Rehabilitation Journal as their official journal. As an online, open-access journal, European Rehabilitation Journal will publish one volume per year, with papers added to the volume online as soon as they are accepted for publication. This will permit rapid publication of papers after acceptance because there will be no need to wait for the next available issue. European Rehabilitation Journal will follow the quality guidelines recommended by the Committee on Publication Ethics [2] and International Society of Physiotherapy Journal Editors [1]. It will use a double-blind peer-review process, publish full-text English language papers, and have low fees sufficient only to support the technical maintenance of the website and help to finance and promote research projects. All original research in European Rehabilitation Journal will be published under a creative commons license, which means that the authors retain ownership of the content and allow the free distribution of it. European Rehabilitation Journal is developed using Open Journal System, which is an open-source solution developed by the Public Knowledge Project [3]; it gathers more than 10,000 scientific, open-access journals and focuses on improving the quality and reach of scholarly publishing.

The editorial board that has been recruited consists of international experts from various fields of research including ageing, cardiorespiratory, intensive care, neurology, pain, paediatrics and sport physiotherapy plus research methodology. Papers in European Rehabilitation Journal are intended to span the gap between research and daily practice, so both fundamental and clinical research will be accepted. The guidelines for authors on the journal's website provide details about the design and structure of the papers. The editorial board also strongly endorses the use of the reporting guidelines on the EQUATOR network by submitting authors.

One of the goals of European Rehabilitation Journal is to be indexed in PubMed within the next few years. This will be achieved by demonstrating high-quality processes and a steady supply of papers with robust methodology and important research findings. While working toward this indexing status, each paper will get a digital object identifier 
(DOI), Crossref will be applied, and ORCID identifiers will be included for authors.

Establishing European Rehabilitation Journal is just one of the many valuable initiatives of the Société Française de Physiothérapie. Members of the Société have already published high quality physiotherapy research in many different journals including Chest, BMJ, Journal of Physiotherapy, Gait \& Posture, Annals of Physical and Rehabilitation Medicine, and Journal of Biomechanics. Members of the Société are also achieving postgraduate qualifications in all areas of rehabilitation and related disciplines such as neurosciences, health economics and engineering. Every year, the Société is enriched by more members who have Masters and $\mathrm{PhD}$ qualifications, further contributing to the evolution of the profession. To further foster research, the Société has instituted academic scholarships and research prizes [4]. In addition, the Société organises scientific conferences every two years to provide opportunities for research dissemination, academic collaboration, and crucial feedback for junior researchers [5] and has collaborated to produce the French section of the Physiotherapy Evidence Database website (PEDro; https://pedro.org.au/french) and French version of the PEDro scale (https://pedro.org.au/french/resources/pedroscale/). The Société is also involved in the recent changes of the status of physiotherapy at the university level. France Mourey, who is an active member of the Société, has been elected President of the Rehabilitation Section (Section 91) in the National University Council (CNU) [6].

World Physiotherapy recommends that training for entry-level physiotherapists should be based on university studies of a minimum of four years, independently validated and accredited as being at a standard that affords graduates full statutory and professional recognition [7]. The Société shares that vision, encouraging its members to take up academic positions. World Physiotherapy provides support and resources to countries where the profession is seeking to establish entry-level physiotherapist education programs at universities [8]. World Physiotherapy's support includes assistance to national physiotherapy associations with the development of appropriate educational standards and with the development of accreditation/recognition processes [9].

We recognise that the initiatives of the Société Française de Physiothérapie are generating excellent conditions for the training of physiotherapists in France to progress to a university degree course, including qualified academic staff with clinical expertise, research skills and publication experience. The 'universitisation' of physiotherapist training will be a crucial step in improving the clinical practice of French physiotherapists and thereby achieve better health outcomes for the French population. And importantly, high-quality clinical research relevant to physiotherapy practice is growing rapidly. At the time of writing, PEDro (https://pedro.org.au) contained over 49,000 trials, reviews and guidelines evaluating physiotherapy interventions [10] and the Diagnostic Test Accuracy database (DiTA; https://dita.org.au) contained nearly 2,000 primary studies and reviews evaluating the accuracy of diagnostic tests used by physiotherapists [11]. This constitutes enormous content for an evidence-based curriculum in physiotherapy.

Internationally, the high academic standards of physiotherapists are receiving greater recognition. For example, the Cochrane Collaboration has appointed a physiotherapist as the co-chair of the Cochrane governing board and another physiotherapist as the co-author of the Cochrane Handbook of Systematic Reviews. Physiotherapists have been appointed to the Organisation for Economic Co-operation and Development (OECD), the World Health Organization, and the Global Rehabilitation Alliance. Physiotherapy in France can share in this success if it becomes an academic discipline. We encourage all movement towards this goal.

\section{References}

[1] International Society of Physiotherapy Journal Editors (ISPJE), 2020. URL https://world.physio/networks/journal-editors.

[2] Search resultsGuidelines / COPE: Committee on Publication Ethics, 2020. URL https://publicationethics.org/guidance/Guidelines.

[3] Public Knowledge Project, 2020. URL https://pkp.sfu.ca/.

[4] Société Française de Physiothérapie - Documentation demande de bourses, 2020. URL https://www.sfphysio.fr/gene/main.php? base $=261$ \&alias=agathe.chervel\&doc_view=arborescence.

[5] Société Française de Physiothérapie - Appel à Communication, 2020. URL https://www.sfphysio.fr/gene/main.php?base=5127\&alias= agathe.chervel\&doc_view=arborescence.

[6] CNU - CNU-SANTE, 2020. URL https://www. conseil-national-des-universites.fr/cnu/\#/entite/entiteName/ CNU-SANTE/idChild/36/idNode/4725-4726.

[7] Guideline: Physical therapist professional entry level education, 2020. URL https://world.physio/guideline/entry-level-education.

[8] Guideline: Qualifications of faculty for physical therapist professional entry level education programmes, 2020. URL https://world. physio/guideline/faculty-qualifications.

[9] Policy statement: Education, 2020. URL https://world.physio/ policy/ps-education.

[10] Anne M. Moseley, Mark R. Elkins, Philip J. Van der Wees, and Marina B. Pinheiro. Using research to guide practice: The Physiotherapy Evidence Database (PEDro). Brazilian Journal of Physical Therapy, 24(5):384-391, September 2020. ISSN 1413-3555. doi: 10.1016/j.bjpt.2019.11.002. URL https://www.sciencedirect.com/ science/article/pii/S1413355519309141.

[11] Mark A. Kaizik, Mark J. Hancock, and Robert D. Herbert. A description of the primary studies of diagnostic test accuracy indexed on the DiTA database. Physiotherapy Research International, 25(4):e1871, 2020. ISSN 1471-2865. doi: https://doi.org/10.1002/pri.1871. URL https://onlinelibrary.wiley.com/doi/abs/10.1002/pri.1871._eprint: https://onlinelibrary.wiley.com/doi/pdf/10.1002/pri.1871. 